\title{
LA FUNCION DE EMPAREJAMIENTO AGREGADA DEL MERCADO LABORAL CHILENO
}

\section{THE AGGREGATE MATCHING FUNCTION FOR THE CHILEAN LABOR MARKET}

\section{ROLANDO EINAR PAZ RODRIGUEZ*}

Universidad Alberto Hurtado

\begin{abstract}
The aggregate matching function is estimated for the Chilean labor market with time varying efficiency and correcting for endogeneity bias for the period 1986-2015. The results found are consistent, statistically significant and show the existence of a structural break around the year 2002 and a job market tightness elasticity of 11,68\% before the break and 12,53\% afterwards.
\end{abstract}

Keywords: Matching function, unemployment, vacancies, job finding probability, job market tightness.

JEL Classification: C13, J6, J63, J64, E24.

\section{Resumen}

Se estima la función de emparejamiento para el mercado laboral chileno con eficiencia variante en el tiempo y corrigiendo por sesgo de endogeneidad para el período 1986-2015. Los resultados encontrados son consistentes, significativos y muestran la existencia de un quiebre estructural alrededor

* Magíster en Economía. E-mail: einarpaz@gmail.com.

Trabajo basado en la Tesis realizada por el autor para optar al grado de Master of Arts in Economics otorgado por Georgetown University y al grado de Magíster en Economía de la Universidad Alberto Hurtado.

El autor agradece los comentarios de Mauricio Tejada, Pablo Gonzales y Carlos García. 
del 2002 y una elasticidad de la rigidez del mercado laboral de 11,68\% antes del quiebre y de $12,53 \%$ después del mismo.

Palabras clave: Función de emparejamiento; desempleo; vacantes; probabilidad de encontrar trabajo; rigidez del mercado laboral.

Clasificación JEL: C13, J6, J63, J64, E24.

\section{INTRODUCCION}

La función de emparejamiento, mejor conocida como matching function, es una herramienta de modelización que dentro de la literatura de economía laboral posee una importancia comparable con la función de producción en macroeconomía. En términos simples, es un instrumento de análisis del funcionamiento del mercado de trabajo y las fricciones existentes en él. En efecto, la función de emparejamiento permite modelar y capturar el proceso, por medio de esta la oferta y demanda de trabajo se encuentran en un mercado, por lo que recogen las consecuencias de las heterogeneidades y fricciones existentes en él, del mismo modo que modelos convencionales, pero en términos de pocas variables y de una forma relativamente simple.

En términos generales y de forma análoga a una función de producción típica que combina un conjunto de inputs con un nivel de conocimientos o tecnología para obtener un determinado bien, la función de emparejamiento formaliza la relación entre los inputs del mercado laboral, vacantes y desocupados, a partir de este se obtiene un resultado u output que corresponde al emparejamiento o match, entendido como el flujo de salida del desempleo. Por su parte, la variable equivalente al nivel tecnológico en el contexto de la función de emparejamiento capturará además las potenciales heterogeneidades, fricciones y eficiencia del mercado de trabajo.

Como señalan Petrongolo y Pissarides (2001), las fricciones dentro del mercado laboral pueden surgir por diferentes factores, así como problemas de información, heterogeneidad de los agentes, ausencia de mercados de seguros, congestión, movilidad lenta de los flujos de entrada y salida del empleo, entre otros. Modelar cada uno de estos aspectos sería una tarea compleja, sin embargo, mediante la función de emparejamiento es posible capturar, en una relación de equilibrio, los efectos de dichas fricciones sin incluirlas de forma explícita.

En la literatura empírica, la función de emparejamiento habitualmente es utilizada como un insumo en distintos tipos de modelos, cumpliendo el papel de herramienta de modelización que recoge las consecuencias de las particularidades del mercado de trabajo. Asimismo, también es utilizada como una herramienta independiente cuya estimación permite el estudio per se de las heterogeneidades, fricciones y externalidades presentes en el mercado laboral. En este sentido, la estimación de la función de emparejamiento es de gran importancia para entender el funcionamiento 
del mencionado mercado y a su vez permitir la elaboración de recomendaciones de política económica adecuadas y oportunas.

Aunque a partir del trabajo seminal de Pissarides (1986) es posible encontrar un gran número de estimaciones de esta función para diversas economías, no existen muchas investigaciones empíricas aplicadas a países latinoamericanos. Esto se debe fundamentalmente a la poca disponibilidad o inexistencia de series temporales confiables acerca de las variables a ser utilizadas, en particular respecto del stock de vacantes y al flujo de salida del desempleo o emparejamientos.

Para el caso chileno, si bien no se cuenta con una serie correspondiente al flujo de emparejamientos, existe una proxy para el stock de vacantes en la forma de un índice de vacantes, que sigue la metodología establecida en el trabajo de Belani, García y Pastén (2002) y que es publicado y actualizado por el Banco Central de Chile. La mencionada investigación utiliza el índice de vacantes para estimar la curva de Beveridge ${ }^{1}$ y deja claro la imposibilidad de estimar de forma directa la función de emparejamiento para Chile, a causa de la falta de información del flujo de salida del desempleo. Sin embargo, los autores estiman una aproximación a la función de emparejamiento para el período 1986-2002, de forma que su trabajo se convierte en el único referente al respecto.

En este sentido, el presente trabajo pretende atender a la necesidad de contar con una estimación apropiada de la función de emparejamiento del mercado laboral chileno que aporte en el entendimiento de su funcionamiento, características y la evaluación de su eficiencia, fricciones y externalidades. Para tal efecto y debido a la limitación en los datos disponibles, se propone la estimación de una transformación equivalente de la función de emparejamiento, misma que utiliza como insumos una variable entendida como la probabilidad de que un desempleado encuentre trabajo (job finding probability), construida siguiendo la metodología propuesta por Shimer (2012), y el ratio entre el índice de vacantes mencionado anteriormente y el stock de desocupados publicado por el Instituto Nacional de Estadística de Chile (INE).

Asimismo, considerando la literatura reciente en términos de aportes teóricos y nuevas aproximaciones empíricas, el presente trabajo estima la función de emparejamiento siguiendo la metodología propuesta por Borowczyk-Martins, Jolivet y Postel-Vinay (2013), quienes utilizan un modelo que corrige un sesgo de endogeneidad habitual en este tipo de investigaciones y toman la eficiencia del emparejamiento como un componente endógeno al modelo. De esta forma se espera contribuir a la literatura empírica con resultados que correspondan a un reflejo fidedigno de la realidad actual del mercado de trabajo chileno y sirvan como insumo en futuras modelizaciones del mismo.

1 La curva de Beveridge, desarrollada en 1958, muestra la relación entre las tasas de desempleo y de vacancias laborales. 
El presente documento está organizado de la siguiente manera: la sección 2 presenta una revisión de la literatura relacionada, la sección 3 describe el marco conceptual detrás de la función de emparejamiento y de su estimación, la sección 4 describe los datos y presenta los resultados de estimación. Finalmente, la sección 5 concluye.

\section{REVISION DE LA LITERATURA}

En la literatura empírica, la función de emparejamiento suele ser estimada como una función agregada para el estudio de heterogeneidades, fricciones y externalidades presentes en el mercado laboral o también es utilizada como una herramienta de modelización simple a manera de insumo en diferentes tipos de modelos. En este sentido, esta sección presenta una revisión de los trabajos de investigación que estiman la función de emparejamiento desde la primera perspectiva señalada anteriormente.

Pissarides (1986), uno de los pioneros en esta literatura, estima una función de emparejamiento agregada para el Reino Unido en el marco de un modelo search and matching. Para ello utiliza los métodos de Mínimos Cuadrados Ordinarios y Variables Instrumentales y asumiendo rendimientos constantes a escala encuentra que la elasticidad del stock de desocupados sobre el flujo de emparejamientos alcanza 70\%. En esta misma línea, Blanchard y Diamond (1989) estiman una función de emparejamiento agregada para Estados Unidos siguiendo la misma metodología, pero también añadiendo estimaciones por Mínimos Cuadrados No Lineales. En dicho trabajo se resalta la analogía entre la función de producción y la función de emparejamiento, así como la existencia de fricciones provocadas por la situación geográfica, heterogeneidades entre trabajadores y trabajos, y que cambios en el parámetro correspondiente a la eficiencia del emparejamiento capturan variaciones en dichas fricciones. En las diferentes especificaciones estimadas, estos autores encontraron evidencia que respalda la presencia de rendimientos constantes y ligeramente crecientes a escala, con elasticidades de desocupados y vacantes sobre el flujo de emparejamientos que fluctúan entre 35 a $67 \%$ y 54 a $70 \%$, respectivamente.

Por su parte, Anderson y Burgess (1995) realizan una estimación de la función de emparejamiento para Estados Unidos usando datos desagregados a nivel estatal e industrial. Utilizando Datos de Panel con Efectos Fijos, los resultados de los autores respaldan la hipótesis de rendimientos ligeramente crecientes a escala, con elasticidades de desocupados y vacantes de alrededor de 45 y $80 \%$, respectivamente. Adicionalmente, argumentan que resultados encontrados anteriormente por otros autores respecto de funciones de emparejamiento agregadas tenían problemas de especificación como resultado de las aproximaciones empleadas en la construcción de las series de tiempo agregadas que utilizaron.

Posteriormente, otro de los grandes aportes a la estimación empírica de la función de emparejamiento fue realizado por Coles y Smith (1998), quienes estiman la función para Gran Bretaña. Estos autores argumentan que, si los desocupados cuentan con información perfecta acerca de las vacantes disponibles, entonces todos ellos se 
postularán a las vacantes que ellos crean convenientes. De esta forma, la existencia de desempleados y vacantes que no pudieron emparejarse se deberá únicamente a que no encontraron a su par apropiado. Así, estas vacantes y desocupados, al inicio del siguiente período, no intentarán emparejarse entre sí, de modo que en ambos casos se tratarán de emparejar con el nuevo flujo de entrada de desocupados y vacantes, respectivamente.

Este supuesto, denominado emparejamiento stock-flow, se ve reflejado en la inclusión de variables flujo para vacantes y desocupados como variables explicativas adicionales en la función de emparejamiento. En este contexto no rechazan la existencia de rendimientos constantes a escala y determinan que la elasticidad de desocupados alcanza 34\%. En esta misma línea, Coles y Petrongolo (2008) también realizan una estimación para Gran Bretaña bajo el enfoque stock-flow, con resultados similares respecto de los retornos a escala de la función, pero con una elasticidad de desocupados de alrededor del $50 \%$. Algunas investigaciones posteriores que siguen esta misma línea son las de Galuscak y Münich (2007) para República Checa, y Alvarez, Núñez y Usabiaga (2008) para España.

Es importante destacar que la gran mayoría de las investigaciones empíricas consideran a la eficiencia del proceso de emparejamiento como una constante con el paso del tiempo. Sin embargo, intuitivamente es natural pensar que la eficiencia del emparejamiento en el mercado laboral de una economía puede sufrir cambios como consecuencia de diferentes sucesos coyunturales o estructurales que puedan ocurrir, como la implementación de políticas o la utilización de tecnologías que modifiquen las heterogeneidades y fricciones en dicho mercado.

$\mathrm{Al}$ respecto, se revisaron algunas investigaciones que sí consideran a la eficiencia del emparejamiento como un componente endógeno o variante con el tiempo. Por ejemplo, tanto Kano y Otha (2005), como Sasaki (2011), estiman funciones de emparejamiento para Japón identificando a la eficiencia del emparejamiento como un componente variante entre regiones, por lo que asumen que hay una eficiencia distinta en cada una de las prefecturas de Japón. Sin embargo, asumen que dicha eficiencia se mantiene constante con el tiempo, por lo que estiman la función con datos de panel y efectos fijos.

Por su parte, Barnichon y Figura (2015) estiman una función de emparejamiento para Estados Unidos, considerando la eficiencia del emparejamiento como un componente endógeno al modelo, por lo que modelan explícitamente heterogeneidades de los trabajadores y segmentación de mercado. En este contexto, los autores identifican un comportamiento procíclico en la eficiencia del emparejamiento con el tiempo y una importante pérdida de eficiencia después del 2007. La elasticidad para la rigidez del mercado laboral, o job market thightness, cae de $33 \%$ a $18 \%$ después del mencionado año.

Finalmente, Borowczyk-Martins et al. (2013) estiman una función de emparejamiento también para Estados Unidos considerando una eficiencia del emparejamiento variante en el tiempo. Estos autores argumentan que levantar el supuesto de eficiencia constante en el tiempo genera un potencial sesgo de endogeneidad en la estimación de funciones 
de emparejamiento agregadas. Este sesgo es provocado por el comportamiento optimizador de las firmas bajo el supuesto de libre entrada y salida de firmas en el mercado laboral, el que es un supuesto habitual en este tipo de investigaciones. La solución propuesta por los autores consiste en considerar a la eficiencia del emparejamiento como un componente inobservado, para este se establece una estructura temporal a ser determinada y estimar la función por MGM instrumentalizando las variables endógenas del modelo. Siguiendo dicha metodología y tomando el supuesto de rendimientos constantes a escala, encuentran que la elasticidad de la rigidez del mercado laboral americano es de 70,6\%.

Como se adelantaba en la sección introductoria, la literatura empírica correspondiente a la estimación de la función de emparejamiento del mercado laboral chileno se ve representada únicamente por el trabajo de Belani et al. (2002). Aunque el objetivo principal de su investigación es presentar un índice de vacantes a nivel nacional para Chile y estimar una curva de Beveridge, también efectúan una aproximación de la función de emparejamiento para el período 1986-2002 usando un modelo de vectores de corrección de errores. Al respecto, los autores suponen rendimientos constantes a escala en la función y sus resultados indican que la elasticidad de vacantes en el flujo de emparejamientos alcanza a $16 \%$.

En este sentido, considerando la literatura revisada, los distintos avances y aportes en lo referente a la estimación empírica de la función de emparejamiento, en la presente investigación se decidió seguir el procedimiento utilizado por Borowczyk-Martins et al. (2013), debido a que es el que más se aproxima al objetivo del presente trabajo.

\section{MARCO CONCEPTUAL}

Tomando en cuenta la definición de la función de emparejamiento:

$$
M=A m(U, V)
$$

Donde la interacción de las vacantes $V$, los desempleados $U$ y el factor tecnológico, heterogeneidades, fricciones y eficiencia del mercado de trabajo $A$, da como resultado el flujo de salida del desempleo, emparejamiento o match $M$. Al respecto, es posible identificar algunos aspectos importantes directamente relacionados con la estimación empírica de la función de emparejamiento, como ser la forma funcional y el tipo de rendimientos.

Respecto de la forma funcional, generalmente se asume que la función de emparejamiento toma la forma de una función Cobb Douglas, como se observa a continuación:

$$
M_{t}=A U_{t}^{\beta_{1}} V_{t}^{\beta_{2}}
$$


A partir de dicha expresión es posible identificar fácilmente las externalidades que pueden producirse en el mercado laboral. Es decir, que las acciones que toman las firmas para llenar sus vacantes influirán en el tiempo que tarda un desempleado en encontrar trabajo, dando lugar a externalidades positivas de vacantes en desempleados. Por otra parte, el hecho que una vacante sea ocupada no solo dependerá del empleador, sino también de las acciones que tomen los desempleados por encontrar trabajo, dando lugar a externalidades positivas de desempleados en vacantes. Sin embargo, las acciones de los desempleados también podrían influir de forma negativa en la búsqueda de trabajo de otros desempleados y algo similar sucede con las vacantes de las firmas.

En este sentido, podemos interpretar a $\beta_{1}$ como una medida de la externalidad positiva de desempleados en vacantes y $\beta_{1}-1$ como la externalidad negativa o congestión entre los desempleados. Asimismo, $\beta_{2}$ será la externalidad positiva de vacantes en desempleados, mejor conocida como thick market effect y $\beta_{2}-1$ será la externalidad negativa de unas vacantes en otras.

Aplicando logaritmos a (2) tendremos una expresión linealizada fácil de estimar empíricamente:

$$
\ln M_{t}=\beta_{0}+\beta_{1} \ln U_{t}+\beta_{2} \ln V_{t}
$$

Siendo $\beta_{0}=\ln A$. Es importante mencionar que algunos autores deciden utilizar alguna variante de la función de emparejamiento Cobb Douglas linealizada, como por ejemplo la función conocida como Trasnlog, como en el caso de Yashiv (2000):

$$
\ln M_{t}=\beta_{0}+\beta_{1} \ln U_{t}+\beta_{2} \ln V_{t}+\left(\frac{1}{2}\right) \beta_{3}\left(\ln U_{t}\right)^{2}+\beta_{4}\left(\ln V_{t}\right)^{2}+\beta_{5}\left(\ln U_{t}\right)\left(\ln V_{t}\right)
$$

Por otra parte, otro aspecto fundamental a tomar en cuenta en la estimación empírica será el tipo de rendimientos a escala de la función. En particular, Si $\beta_{1}+\beta_{2}=1$ la función muestra rendimientos constantes a escala, si $\beta_{1}+\beta_{2}>1$ los rendimientos serán crecientes a escala y finalmente si $\beta_{1}+\beta_{2}<1$ serán rendimientos decrecientes a escala. En la literatura empírica es posible encontrar estudios que respaldan uno u otro tipo de rendimientos a escala, variando incluso para un mismo país.

En general, la importancia respecto de los rendimientos a escala surge en situaciones en las que la función de emparejamiento es utilizada como herramienta de modelización. Por ejemplo, en el contexto de una versión simplificada del modelo de desempleo de equilibrio de Pissarides (2000), estando la función de emparejamiento contenida en dicho modelo y asumiendo rendimientos constantes a escala, el equilibrio resultante es único, estable y óptimo, por lo que no hay la necesidad de intervención estatal en el proceso de emparejamiento. Sin embargo, en presencia de rendimientos crecientes a escala, el equilibrio no es único, por lo que podría justificarse la intervención estatal con el objetivo de llevar a la economía al equilibrio con más bajo nivel de desempleo. 
De las investigaciones revisadas, la gran mayoría de ellas utiliza para la estimación de la función de emparejamiento la forma funcional Cobb Douglas linealizada con rendimientos constantes a escala. Esta investigación sigue esta misma línea, utilizando las mencionadas características, y siguiendo a Borowczyk-Martins et al. (2013) se considera a la eficiencia del emparejamiento como un componente que potencialmente puede variar en el tiempo, por lo que la función a estimar es la siguiente:

$$
M_{t}=A_{t} V_{t}^{\alpha} U_{t}^{1-\alpha}
$$

Donde $M_{t}$ corresponde al flujo de emparejamientos, $A_{t}$ será la eficiencia del emparejamiento variante con el tiempo, $V_{t}$ las vacantes y finalmente $U_{t}$ el stock de desempleados.

Siguiendo nuevamente a Borowczyk-Martins et al. (2013) y tomando en cuenta que en Chile no se cuenta con información acerca del flujo de emparejamientos $M_{t}$, es necesario hacer una transformación en la función propuesta, de forma que se pueda utilizar otra variable alternativa que sí esté disponible o que se pueda construir. En este sentido, dividiendo (5) entre $U_{t}$ tendremos:

$$
\underbrace{\frac{M_{t}}{U_{t}}=A_{t}}_{F_{t}=} A_{t} \underbrace{\left(\frac{V_{t}}{U_{t}}\right)^{\alpha}}_{\Theta_{t}^{\alpha}}
$$

Donde el cociente $\left(M_{t} / U_{t}\right)=F_{t}$ se entiende como la probabilidad de salida del desempleo o la probabilidad que tiene un desempleado de encontrar trabajo; $\Theta_{t}$ a su vez, es una medida de rigidez del mercado laboral y finalmente $A_{t}$ continúa siendo la eficiencia del emparejamiento. Nótese que para realizar esta transformación es necesario suponer que la función de emparejamiento presenta rendimientos constantes a escala.

La nueva función de emparejamiento transformada (6) está conformada por variables disponibles o de cálculo relativamente sencillo, haciendo viable su implementación empírica. Más aún, aplicando logaritmos se tiene la especificación habitual de la literatura. Sin embargo, como Borowczyk-Martins et al. (2013) destacan, suponer que la eficiencia del emparejamiento puede cambiar en el tiempo como respuesta a shocks introduce un problema de sesgo de endogeneidad en la estimación.

En particular, la endogeneidad surge por el comportamiento optimizador de las firmas en el proceso de búsqueda de trabajadores. Tomando el supuesto de libre entrada y salida de firmas en el mercado laboral, las mismas igualarán el costo marginal de buscar trabajadores para sus vacancias disponibles con el beneficio esperado de dicho puesto de trabajo ocupado, por lo que siempre que el costo sea menor o igual al beneficio esperado, las firmas seguirán buscando trabajadores. Dicha relación puede representarse de la siguiente forma: 


$$
C_{t}=\frac{M_{t}}{V_{t}} \Pi_{t}
$$

Donde $C_{t}$ es el costo marginal de publicar una vacancia para la firma, $\Pi_{t}$ es el valor presente descontado del puesto de trabajo ocupado, multiplicado por la probabilidad de que un trabajo se ocupe, que desde la perspectiva de la firma es equivalente a $M_{t} / V_{t}$. Luego, tomando la función de emparejamiento original (5) y dividiéndola entre $V_{t}$ tendremos la siguiente expresión:

$$
\frac{M_{t}}{V_{t}}=A_{t} \Theta_{t}^{\alpha-1}
$$

Reemplazando (8) en (7) y despejando $\Theta$ tendremos:

$$
\Theta_{t}^{1-\alpha}=\frac{A_{t} \Pi_{t}}{C_{t}}
$$

De la ecuación (9) se hace evidente que existe simultaneidad entre la rigidez del mercado laboral $\Theta_{t}$ y la eficiencia del emparejamiento $A_{t}$, estando ambas correlacionadas $\operatorname{Cov}\left(\Theta_{t}, A_{t}\right) \neq 0$. En este contexto, cualquier shock sobre la eficiencia del emparejamiento generará un cambio en el número de emparejamientos y con ello (y de forma indirecta) el número de vacancias óptima. Esto implica que la rigidez de mercado (de la cual forma parte el número de vacancias) es una variable endógena. Por lo tanto, cualquier tipo de estimación por Mínimos Cuadrados Ordinarios de la elasticidad $\alpha$ correspondiente a la función (6) linealizada estará entonces sesgada.

La estrategia propuesta por Borowczyk-Martins et al. (2013) consiste en imponer cierta estructura a la eficiencia del emparejamiento y transformar el modelo en base a dicha estructura para luego estimarlo por el Método Generalizado de Momentos (MGM). Por lo tanto, tomando la función de emparejamiento (6), aplicando logaritmos y definiendo las variables logaritmizadas en minúsculas, la función resultante será:

$$
f_{t}=a_{t}+\alpha \theta_{t}
$$

Luego, se propone la siguiente estructura para la eficiencia del emparejamiento:

$$
a_{t}=\mu+\epsilon_{t}
$$

Donde $\mu$ corresponde a un componente constante de la eficiencia y $\epsilon_{t}$ será un componente estocástico inobservado. Reemplazando (11) en (10) se tiene:

$$
f_{t}=\mu+\alpha \theta_{t}+\epsilon_{t}
$$


A continuación, se asu me que el componente inobservado de la eficiencia del emparejamiento $\epsilon_{t}$ sigue un proceso $\operatorname{ARMA}(p, q)$ estacionario que toma la siguiente forma:

$$
\epsilon_{t}=\sum_{\ell=1}^{p} \rho_{\ell} \epsilon_{t-\ell}+\sum_{\ell=1}^{q} \alpha_{\ell} \omega_{t} \Leftrightarrow P(L) \epsilon_{t}=Q(L) \omega_{t}
$$

Donde $L$ es el operador rezago, $\omega_{t}$ es ruido blanco y $P(L)=1-\sum_{\ell=1}^{p} \rho_{\ell} L^{\ell}$. Aplicando la transformación $P(L)$ a (12) y tomando en cuenta lo definido para el proceso estocástico $\epsilon_{t}$, se tiene:

$$
f_{t}=\gamma+\sum_{\ell=1}^{p} \rho_{\ell} f_{t-\ell}+\alpha \theta_{t}-\sum_{\ell=1}^{p} \lambda_{\ell} \theta_{t-\ell}+Q(L) \omega_{t}
$$

Con $\gamma=\left(1-\sum_{\ell=1}^{p} \rho_{\ell}\right) \mu$ y para todo $\ell$ tendremos que $\lambda_{\ell}=\alpha \rho_{\ell}$. Por tanto, la ecuación (10) es la especificación final del modelo a estimar usando MGM. A causa del sesgo de endogeneidad identificado anteriormente, y debido a la persistencia del componente $M A(q)$ de $\epsilon_{t}$, todos los regresores $\left(\theta_{t}, \ldots, \theta_{t-\min \{p, q\}}\right)$ y si $q \geq 1$, también $\left(f_{t-1}, \ldots, f_{t-\min \{p, q\}}\right)$ serán endógenos. En consecuencia, considerando $\alpha$ y los coeficientes autorregresivos $\rho_{\ell}$, tenemos un total de $p+1$ parámetros a ser estimados (ignorando las variables exógenas como la constante o variables dicótomas que pueden ser utilizadas como instrumentos para sí mismas), que equivaldrá al número de condiciones de momento necesarias para la identificación.

En este sentido, rezagos tanto de $\theta_{t}$ y $f_{t}$ superiores al orden del proceso $M A(q)$ pueden ser usados como instrumentos válidos, toda vez que la relación de simultaneidad entre la eficiencia del emparejamiento y la rigidez del mercado laboral estaría presente solamente hasta el rezago $q$ en la función transformada (14). Así, la estimación estructural por MGM en este caso descansa sobre las condiciones de momentos $E\left(\omega_{t}, \theta_{t-\ell}\right)=0$ y $E\left(\omega_{t}, f_{t-\ell}\right)=0$ para todo $\ell \geq q+1$, siendo variables que no tendrían un efecto directo sobre $f$ en el período $t$, pero que sin embargo están relacionadas con los regresores endógenos. Es interesante mencionar que bajo este precepto existe la posibilidad de sobreidentificar el modelo.

Para determinar el orden del proceso ARMA correspondiente al componente inobservado de la eficiencia del emparejamiento, Borowczyk-Martins et al. (2013) proponen una metodología de estimación, que, aunque informal, es bastante intuitiva y fácil de implementar en la práctica. Primero se estima el modelo definido por la ecuación (14) para un grid de valores de $p \in(1, \ldots, \bar{p})$ correspondientes al componente autorregresivo. Para esta investigación se definió $\bar{p}=6$ debido a que rezagos superiores no son significativos en ningún contexto. Luego se selecciona el valor más alto de 
$p$ para el que se estiman coeficientes autorregresivos estadísticamente significativos $\left(\rho_{\ell}\right)$. A continuación se estima nuevamente el modelo con el valor $p$ seleccionado anteriormente, pero esta vez para un grid de valores similar $q \in(0, \ldots, \bar{q})$, definiéndose el valor de $q$ por la inspección del autocorrelograma de residuos. El modelo más adecuado será aquel que no muestre un nivel de autocorrelación estadísticamente significativo superior al orden $q$.

Como se mencionó anteriormente, los rezagos de $\theta_{t}$ o $f_{t}$ mayores o iguales a $q+1$, serán instrumentos válidos para $\left(\theta_{t}, \ldots, \theta_{t-\min \{p, q\}}\right)$ y $\left(f_{t}, \ldots, f_{t-\min \{p, q\}}\right)$, sin mencionar a la constante que es exógena y funciona como instrumento para sí misma. Esta metodología también permite verificar la sensibilidad de la estimación de la elasticidad $\alpha$ a diferentes niveles de persistencia de shocks a la eficiencia del emparejamiento, así como verificar la robustez de los resultados.

\section{RESULTADOS}

\subsection{Datos}

Para la información referente a las vacantes se utilizó el índice trimestral de vacantes de trabajo publicado por el Banco Central de Chile, el mismo que se encuentra disponible desde el primer trimestre de 1986 al cuarto de $2015^{2}$. Este índice es elaborado basado en los avisos de ofertas de trabajo publicados los días domingos en los periódicos más importantes de las cinco zonas urbanas más pobladas del país: El Mercurio de Antofagasta, Valparaíso y Santiago; El Sur de Concepción y El Diario Austral de La Araucanía. La serie total ponderada corresponde a las cifras ajustadas según la información de ocupación por grupo ocupacional y región que entrega el Instituto Nacional de Estadística (INE), dicha metodología corresponde a Belani et al. (2002).

El comportamiento del índice tiene marcadas características estacionales y un comportamiento procíclico. En efecto, como se observa en la Figura 1, existe una tendencia creciente desde el inicio de la serie hasta la llegada de la crisis asiática, durante 1998-1999, y posteriormente se aprecia una recuperación para nuevamente caer durante la crisis subprime del 2008. Finalmente, pese a que en la actualidad la crisis ya fue superada, en los últimos años el índice muestra una clara tendencia decreciente, probablemente debido, entre otras cosas, al auge de otros medios de publicación de vacantes como ser el internet.

Es importante destacar que el índice de vacantes, aunque representa un proxy adecuado para la totalidad de vacantes disponibles, también adolece de algunos problemas que podrían afectar su adecuada cuantificación. Mujica (2007) destaca los dos principales problemas que posee el índice. Primero, el índice excluye las vacantes

2 Más específicamente, se tomó en cuenta la serie original del índice total de vacantes ponderado. 


\section{FIGURA 1}

INDICE DE VACANTES TRIMESTRAL PONDERADO, 1986-2015

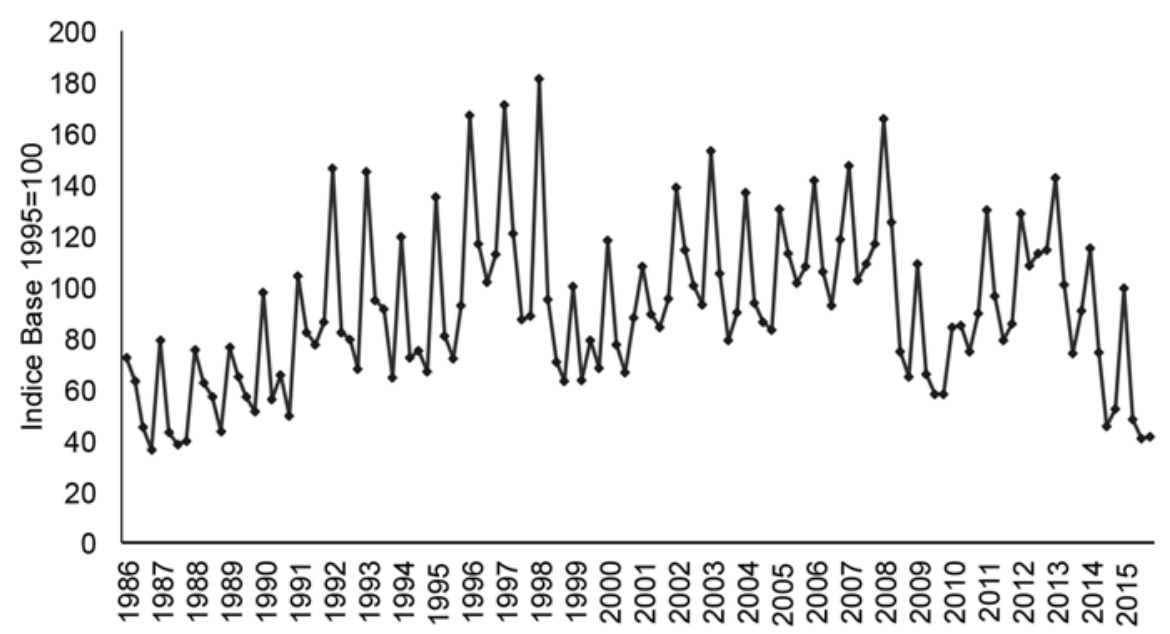

Fuente: Banco Central de Chile.

de los sectores agrícola y construcción, esto debido a que las vacantes ofrecidas en esos sectores no son publicadas regularmente en los periódicos. Segundo, no incluye las vacantes correspondientes a otros medios alternativos de publicación, como las agencias de empleo y principalmente el Internet, medio que se consolida mundialmente como un sustituto de los anuncios en periódicos.

Respecto del stock de desempleados, se utilizó la información trimestral publicada por el Instituto Nacional de Estadística (INE). Para 1986 a 2009 se utilizan las series empalmadas por el INE con base en Censo 2002 provenientes de la Encuesta Nacional de Empleo (ENE). Desde el primer trimestre de 2010 en adelante se usan las series obtenidas a partir de la Nueva Encuesta Nacional de Empleo (NENE).

La ENE vigente desde 1966 hasta 2009 y la NENE puesta en funcionamiento desde 2010 al presente, son encuestas continuas, que generan resultados para trimestres móviles, están diseñadas para entregar resultados a nivel nacional y regional y cuentan con representatividad para cada una de las 15 regiones de Chile.

La implementación de los cambios a la encuesta de empleo llevados a cabo a partir de la NENE siguieron las recomendaciones y exigencias realizadas por organismos internacionales como la Organización Internacional del Trabajo (OIT), la Organización para la Cooperación y Desarrollo Económico (OCDE) y la Oficina de Estadísticas Laborales de EE.UU. La NENE como encuesta a hogares de carácter continuo y basada en una muestra trimestral de aproximadamente 36.000 hogares en 
todo el país, incorpora actualizaciones metodológicas y aporta nueva información respecto de las características del empleo y acerca de las personas inactivas o fuera de la fuerza de trabajo.

Como se observa en la Figura 2, la serie correspondiente al stock de desempleados presenta cierto componente estacional, pero lo que más destaca son algunos saltos significativos principalmente alrededor de 1997 y 2009, lo que concuerda con las recesiones más importantes que tuvo Chile en los últimos 30 años. Destaca también que la recuperación del mercado laboral, en forma de una reducción en el número de desempleados, después de la crisis asiática de 1998, es marcadamente diferente a lo observado después de la crisis subprime de 2008.

En efecto, el desempleo posterior a la crisis de 1998 empieza a caer de forma evidente solo a partir de 2004, seis años después de la recesión, mientras que en 2008 la recuperación es casi instantánea. Es probable que la marcada caída en el desempleo en 2010 y 2011 sea resultado de los efectos del terremoto de febrero 2010, no obstante, queda la duda de si efectivamente ha ocurrido un cambio en el ajuste del mercado laboral. Este elemento es considerado posteriormente en la estimación del modelo final.

Finalmente, el ratio correspondiente a $F_{t}=M_{t} / U_{t}$ entendido como la probabilidad que una persona desempleada encuentre trabajo, fue obtenida a base de la metodología propuesta por Shimer (2012). El autor reevalúa las entradas y salidas del desempleo para Estados Unidos sobre la base de la información correspondiente a número de

FIGURA 2

STOCK DE DESOCUPADOS TRIMESTRAL, 1986-2015

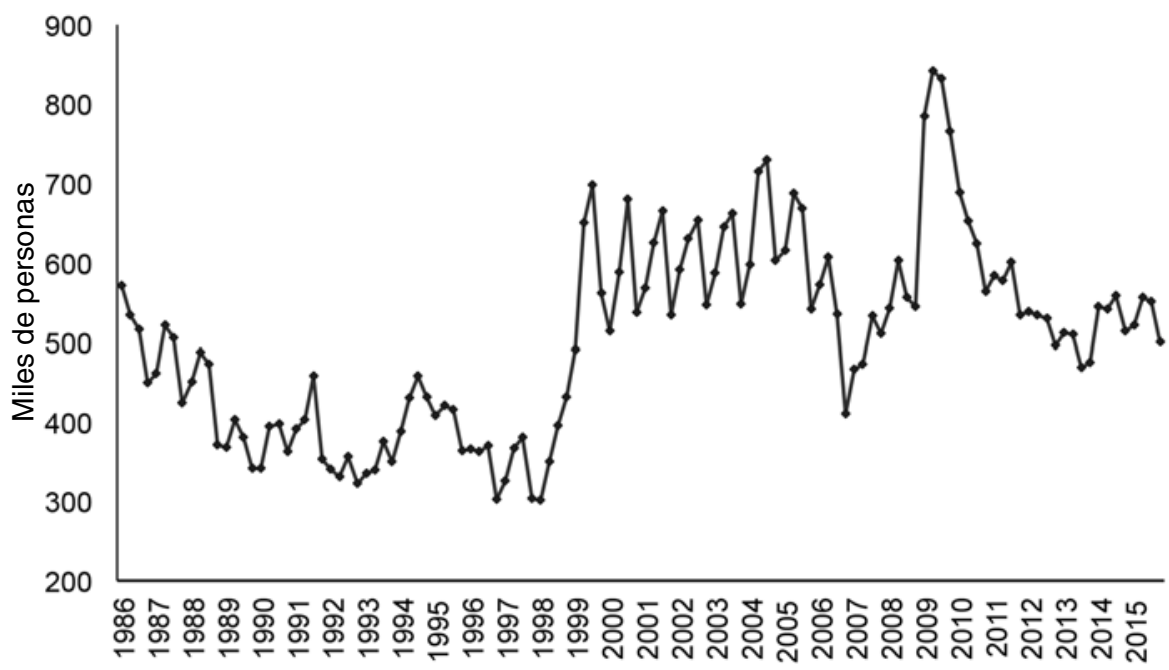

Fuente: INE. 
desempleados, el número de empleados y la duración del desempleo. A continuación se describe brevemente dicha metodología.

Sea $U_{t}$ el número total de desempleados en el período $t$ y asumiendo que todos los trabajadores desempleados encuentran trabajo en el período $t$ con probabilidad $F_{t}$ (y suponiendo que ningún desempleado sale de la fuerza de trabajo), el número de desempleados del período siguiente, $U_{t+1}$ será igual al número de desempleados en $t$ que no pudieron encontrar trabajo, más los desempleados de corto plazo $U_{t+1}^{s}$, quienes serán aquellos que están desempleados en $t+1$ pero que tuvieron trabajo en algún momento entre los períodos $t$ y $t+1$ :

$$
U_{t+1}=\left(1-F_{t}\right) U_{t}+U_{t+1}^{s}
$$

Despejando $F_{t}$ de (15), tendremos una expresión para la probabilidad de encontrar trabajo en términos del stock de desempleados total y el de corto plazo:

$$
F_{t}=1-\frac{U_{t+1}-U_{t+1}^{s}}{U_{t}}
$$

Shimer (2012), al utilizar datos mensuales, define a $U_{t}^{s}$ como el número de desempleados que buscaron trabajo entre 0 a 4 semanas. Para el caso chileno, es posible obtener $U_{t}^{s}$ a partir de las ENE y NENE, pero definiendo como desempleo de corto plazo al número de desempleados que buscaron trabajo entre 0 a 3 meses. Esta restricción se impone debido a la construcción misma de las ENE y NENE, que están definidas en términos de trimestres móviles.

Para la identificación de los desempleados de corto plazo, en el caso de la ENE, se utilizó la pregunta referente al número de semanas que el desocupado busca trabajo. En el caso de la NENE, y debido a que esta eliminó dicha pregunta del cuestionario, fue necesario calcular el desempleo de corto plazo a partir la información del año y mes de levantamiento de la encuesta y la del año y mes desde el que el desocupado informó que empezó a buscar empleo. Generando la serie correspondiente a $U_{t}^{s}$ y siguiendo la ecuación (16) se obtuvo la probabilidad de encontrar trabajo para el período de interés, como se aprecia en la Figura 3.

Un punto importante a destacar acerca de la metodología propuesta por Shimer (2012), para calcular la probabilidad de encontrar trabajo, es que la misma descansa sobre el supuesto que todos los trabajadores tienen la misma probabilidad de encontrar trabajo. Aunque este supuesto pueda parecer poco realista en la práctica, Shimer (2003) demuestra que en el caso en que los desocupados sean heterogéneos, la probabilidad propuesta siguiendo su metodología corresponde a la media de $F_{t}$ del total de desocupados ${ }^{3}$.

3 Adicionalmente, Shimer (2003) compara su propia medida de $F_{t}$ con otras medidas alternativas, evidenciando que las mismas no difieren de forma importante. 


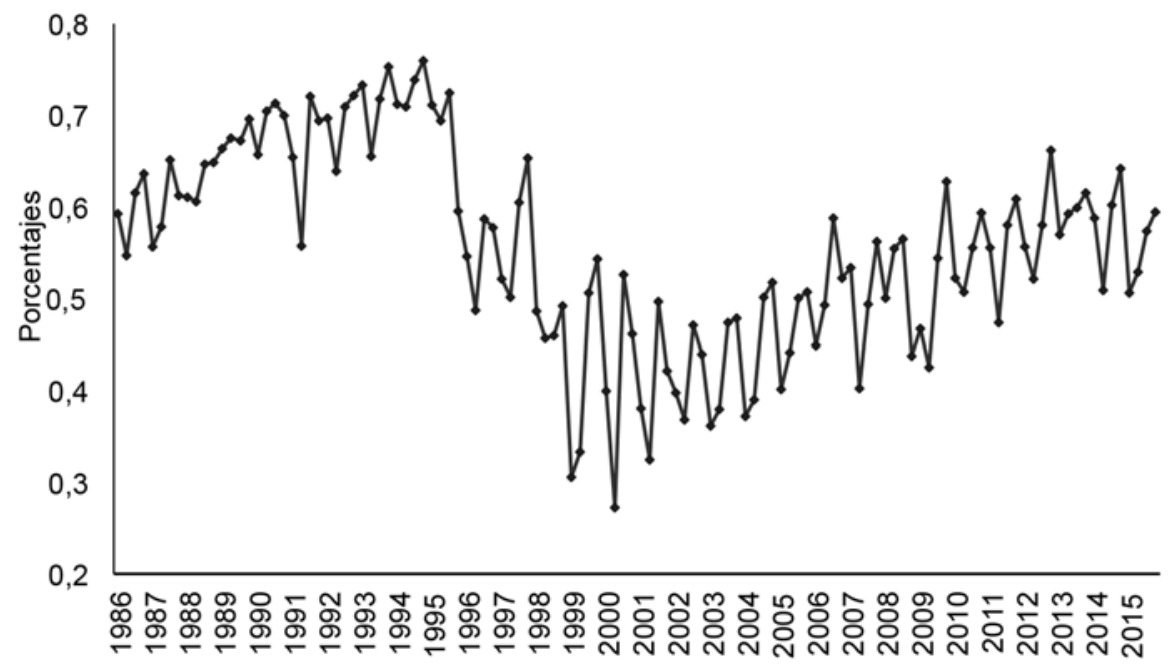

Fuente: Elaboración propia.

En la Tabla 1 se presentan algunas estadísticas descriptivas de las series temporales descritas anteriormente, $\mathrm{y}$ tomando en cuenta la función linealizada a ser reestimada (14), también se añade el ratio correspondiente a la rigidez del mercado laboral $\Theta_{t}=V_{t} / U_{t}$ y sus equivalentes en logaritmos.

Contándose con 120 observaciones, la primera parte de la Tabla 1 corresponde a las estadísticas descriptivas del total de la muestra y adicionalmente se añaden las estadísticas correspondientes a dos submuestras obtenidas a partir de la división del total de observaciones en dos partes, teniéndose 60 observaciones en cada una, como una forma inicial de evidenciar la presencia de algún posible quiebre estructural en alguna de las variables. Al respecto, es posible destacar diferencias significativas en la mayoría de las estadísticas entre submuestras para el stock de desocupados, el índice de vacantes y la probabilidad de encontrar trabajo.

Asimismo, en la Tabla 2 se presentan las correlaciones entre las variables logaritmizadas. La correlación del logaritmo de la probabilidad de encontrar trabajo $f_{t}$ respecto del logaritmo de la medida de rigidez del mercado laboral $\theta_{t}$ alcanza 0,052 respecto del logaritmo de desocupados $-0,542$ y $-0,306$ respecto de logaritmo de vacantes. Por su parte, la correlación del logaritmo de la rigidez del mercado laboral y los logaritmos de vacantes y desocupados es positiva en el primer caso y negativa en el segundo, con 0,833 y - 0,588 , respectivamente. Realizando un análisis extendido 


\section{TABLA 1}

RESUMEN DE ESTADISTICAS DESCRIPTIVAS

\begin{tabular}{|l|r|r|c|c|c|c|c|}
\cline { 2 - 7 } \multicolumn{1}{c|}{} & $U_{t}$ & \multicolumn{1}{c|}{$V_{t}$} & $F_{t}$ & $u_{t}$ & $v_{t}$ & $f_{t}$ & $\theta_{t}$ \\
\hline Media & 505,62 & 90,26 & 0,56 & 13,10 & 4,44 & $-0,61$ & $-8,66$ \\
Mediana & 514,88 & 86,79 & 0,56 & 13,15 & 4,46 & $-0,59$ & $-8,68$ \\
Máximo & 841,89 & 181,25 & 0,76 & 13,64 & 5,20 & $-0,27$ & $-7,42$ \\
Mínimo & 301,31 & 36,15 & 0,27 & 12,62 & 3,59 & $-1,30$ & $-9,62$ \\
Desviación estándar & 120,63 & 31,32 & 0,11 & 0,24 & 0,36 & 0,21 & 0,44 \\
\hline \multicolumn{7}{|c|}{ Submuestra 1 (1986-2000) } \\
\hline Media & 425,60 & 84,49 & 0,60 & 12,94 & 4,37 & $-0,53$ & $-8,57$ \\
Mediana & 397,66 & 77,50 & 0,64 & 12,89 & 4,35 & $-0,45$ & $-8,63$ \\
Máximo & 697,31 & 181,25 & 0,76 & 13,45 & 5,20 & $-0,27$ & $-7,42$ \\
Mínimo & 301,31 & 36,15 & 0,27 & 12,62 & 3,59 & $-1,30$ & $-9,49$ \\
Desviación estándar & 93,12 & 32,30 & 0,12 & 0,20 & 0,37 & 0,22 & 0,48 \\
\hline \multicolumn{7}{|c|}{ Submuestra 2(2001-2015) } \\
\hline Media & 585,65 & 96,03 & 0,51 & 13,27 & 4,51 & $-0,69$ & $-8,76$ \\
Mediana & 557,95 & 95,40 & 0,51 & 13,23 & 4,56 & $-0,68$ & $-8,71$ \\
Máximo & 841,89 & 165,70 & 0,66 & 13,64 & 5,11 & $-0,41$ & $-8,06$ \\
Mínimo & 409,94 & 39,48 & 0,32 & 12,92 & 3,68 & $-1,13$ & $-9,62$ \\
Desviación estándar & 87,49 & 29,45 & 0,08 & 0,14 & 0,34 & 0,16 & 0,39 \\
\hline
\end{tabular}

Fuente: Elaboración propia.

Nota: $\quad$ Stock de desocupados $U_{t}$ en miles de personas.

Variables en minúsculas corresponden a variables logaritmizadas.

TABLA 2

CORRELACIONES ENTRE VARIABLES LOGARITMIZADAS

\begin{tabular}{|c|c|c|c|c|}
\hline & $f_{t}$ & $\theta_{t}$ & $u_{t}$ & $v_{t}$ \\
\hline $\begin{array}{l}f_{t} \\
\theta_{t} \\
u_{t} \\
v_{t}\end{array}$ & $\begin{array}{c}1 \\
0,052 \\
-0,542 \\
-0,306 \\
\end{array}$ & $\begin{array}{c}0,052 \\
1 \\
-0,588 \\
0,833 \\
\end{array}$ & $\begin{array}{c}-0,542 \\
-0,588 \\
1 \\
-0,042 \\
\end{array}$ & $\begin{array}{c}-0,306 \\
0,833 \\
-0,042 \\
1 \\
\end{array}$ \\
\hline \multicolumn{5}{|c|}{ Submuestra 1 (1986-2000) } \\
\hline & $f_{t}$ & $\theta_{t}$ & $u_{t}$ & $v_{t}$ \\
\hline $\begin{array}{l}f_{t} \\
\theta_{t} \\
u_{t} \\
v_{t}\end{array}$ & $\begin{array}{c}1 \\
0,052 \\
-0,436 \\
-0,172\end{array}$ & $\begin{array}{c}0,052 \\
1 \\
-0,716 \\
0,923\end{array}$ & $\begin{array}{c}-0,436 \\
-0,716 \\
1 \\
-0,392\end{array}$ & $\begin{array}{c}-0,172 \\
0,923 \\
-0,392 \\
1\end{array}$ \\
\hline \multicolumn{5}{|c|}{ Submuestra 2 (2001-2015) } \\
\hline & $f_{t}$ & $\theta_{t}$ & $u_{t}$ & $v_{t}$ \\
\hline $\begin{array}{l}f_{t} \\
\theta_{t} \\
u_{t} \\
v_{t}\end{array}$ & $\begin{array}{c}1 \\
-0,173 \\
-0,308 \\
-0,335\end{array}$ & $\begin{array}{c}-0,173 \\
1 \\
-0,505 \\
0,924\end{array}$ & $\begin{array}{c}-0,308 \\
-0,505 \\
1 \\
-0,138\end{array}$ & $\begin{array}{c}-0,335 \\
0,924 \\
-0,138 \\
1\end{array}$ \\
\hline
\end{tabular}

Fuente: Elaboración propia. 
considerando las dos submuestras presentadas en la Tabla 1, observamos que en general los órdenes de magnitud y signos de las correlaciones son muy similares entre submuestras y respecto del total de observaciones.

\subsection{Estacionalidad y raíces unitarias}

A partir de una revisión gráfica de las variables presentadas en las Figuras 1, 2 y 3 , es evidente que las mismas presentan un marcado componente estacional. En este sentido se decidió evaluar a qué clase de proceso generador de datos corresponde la estacionalidad de las variables (proceso estacional determinístico, proceso estacional estacionario o un proceso estacional integrado), con el objeto de aplicar un tratamiento adecuado en la especificación del modelo. Considerando la frecuencia trimestral de los datos, podrían encontrarse hasta dos raíces unitarias estacionales.

Para tal efecto, se utilizó el procedimiento propuesto por Hylleberg, Engle, Granger, y Yoo (1990), comúnmente conocido como test HEGY, cuyos resultados se aprecian en la Tabla 3. La especificación de los componentes determinísticos a ser incluidos en el test para cada variable fueron basados en el análisis gráfico de las mismas, de forma que se incluyeron un intercepto y dummies estacionales en todos los casos ${ }^{4}$.

De acuerdo con los resultados de la prueba HEGY (Tabla 3) aplicados en las series del índice de vacantes, stock de desocupados y la probabilidad de encontrar trabajo, con un nivel de significancia del 5\% se rechaza la hipótesis nula de la presencia de raíces unitarias estacionales de dos y cuatro trimestres por ciclo, habiendo evidencia de que existe una raíz unitaria en la frecuencia cero o no estacional. Por tanto, la forma más adecuada de controlar los efectos estacionales en el modelo a estimar será por medio de la introducción de variables ficticias trimestrales ${ }^{5}$.

Finalmente, considerando la posibilidad de la presencia de raíces unitarias en las variables a ser utilizadas en la estimación de la ecuación (14), se llevó a cabo el test Dickey-Fuller aumentado sobre el logaritmo de la probabilidad de encontrar trabajo $f_{t}$ y sobre el logaritmo de la rigidez del mercado de trabajo $\ln \left(V_{t} / U_{t}\right)=\theta_{t}$. En las pruebas de raíz unitaria se consideraron diferentes especificaciones respecto de los componentes en cada test. Como se observa en la Tabla 4 no se puede rechazar la hipótesis de una raíz unitaria para ambas variables respecto de los valores críticos al $1 \%$. Así, la conclusión en ambos casos es consistente con un proceso $I(1)$, es decir no son estacionarias.

4 Los resultados son robustos a variantes en la especificación de los componentes determinísticos.

5 Borowczyk-Martins et al. (2013) controlan la estacionalidad con variables ficticias y no con series ajustadas por estacionalidad. Esto debido a que el ajuste estacional previo de los datos crearía una correlación serial artificial en todas las variables ajustadas que podría combinarse con el problema de endogeneidad que los autores abordan. 
TABLA 3

TEST HEGY PARA RAICES UNITARIAS ESTACIONALES

\begin{tabular}{|c|l|r|r|}
\hline Variable & \multicolumn{1}{|c|}{ Hipótesis nula } & Estadístico & $\begin{array}{c}\text { Valor crítico } \\
\text { al 5\% }\end{array}$ \\
\cline { 1 - 2 } Indice de & Raíz unitaria no estacional (Frecuencia cero) & $-3,47$ & $-2,03$ \\
vacantes & Raíz unitaria estacional (2 trimestres por ciclo) & $-1,95$ & $-4,87$ \\
$V_{t}$ & Raíz unitaria estacional (4 trimestres por ciclo) & 2,96 & 40,17 \\
\cline { 1 - 2 } Stock de & Raíz unitaria no estacional (Frecuencia cero) & $-3,47$ & $-2,04$ \\
desocupados & Raíz unitaria estacional (2 trimestres por ciclo) & $-1,95$ & $-8,08$ \\
$U_{t}$ & Raíz unitaria estacional (4 trimestres por ciclo) & 2,96 & 33,44 \\
\cline { 1 - 2 } Probabilidad de & Raíz unitaria no estacional (Frecuencia cero) & $-3,47$ & $-1,34$ \\
encontrar trabajo & Raíz unitaria estacional (2 trimestres por ciclo) & $-1,95$ & $-4,89$ \\
$F_{t}$ & Raíz unitaria estacional (4 trimestres por ciclo) & 2,96 & 27,24 \\
\hline
\end{tabular}

Fuente: Elaboración propia.

Nota: Valores críticos correspondientes a Franses y Hobijn (1997).

Selección de rezagos a partir del criterio de Akaike.

TABLA 4

TEST DICKEY-FULLER AUMENTADO

\begin{tabular}{|c|l|l|c|c|}
\hline Variable & \multirow{2}{*}{ Orden de integración } & \multicolumn{1}{|c|}{ Componentes } & Estadístico $\tau$ & $\begin{array}{c}\text { Valor crítico } \\
\text { al } 1 \%\end{array}$ \\
\hline \multirow{4}{*}{$\theta_{t}$} & \multirow{2}{*}{ Primeras diferencias $I(2)$} & Intercepto y tendencia & $-4,21$ & $-4,04$ \\
& & Intercepto & $-3,92$ & $-3,49$ \\
& \multirow{2}{*}{ Niveles $I(1)$} & Intercepto y tendencia & $-3,55$ & $-4,04$ \\
& \multirow{2}{*}{$f_{t}$} & \multirow{2}{*}{ Primeras diferencias $I(2)$} & $-3,06$ & $-3,49$ \\
& \multirow{2}{*}{ Niveles $I(1)$} & Intercepto y tendencia & $-15,49$ & $-4,04$ \\
& & Intercepto & $-15,55$ & $-3,49$ \\
& & Intercepto y tendencia & $-1,12$ & $-4,04$ \\
& Intercepto & $-1,19$ & $-3,49$ \\
\hline
\end{tabular}

Fuente: Elaboración propia.

Nota: Valores críticos correspondientes a MacKinnon (1996).

Aunque la presencia de no estacionariedad pueda sugerir la existencia de una relación espuria entre dichas variables, como señala Hsiao (1997), este problema no está presente en estimaciones realizadas por MGM y otros métodos basados en variables instrumentales, y por tanto dichos métodos generan estimaciones que son consistentes. Lo anterior es clave, ya que el objetivo de la presente investigación es encontrar una relación estructural entre $f_{t}$ y $\theta_{t}$, expresada mediante la elasticidad $\alpha$. 


\subsection{Quiebre estructural}

Considerando las características, comportamiento y diferencias observadas en las estadísticas descriptivas de las series utilizadas entre submuestras (Tabla 1), se realizó un test de quiebre estructural. En este sentido, y considerando que se desconoce la fecha del potencial quiebre, se utilizó la prueba del Ratio de Verosimilitud de Quandt en (12), ignorando el componente inobservado de la eficiencia del emparejamiento $\mathrm{y}$ añadiendo variables ficticias trimestrales $\tau_{i, t}$ para controlar por posibles efectos estacionales, teniéndose el siguiente modelo:

$$
f_{t}=\mu+\alpha \theta_{t}+\sum_{i=1}^{3} \beta_{i} \tau_{i, t}
$$

Se realizó el test del Ratio de Verosimilitud de Quandt usando tres especificaciones para comprobar la existencia de quiebres en el intercepto, en la pendiente y en ambos simultáneamente. En todos los casos los resultados dan evidencia de la existencia de un quiebre estructural.

La Figura 4 muestra el estadístico correspondiente al test de quiebre en intercepto y pendiente simultáneamente. El estadístico supera desde un inicio los valores críticos

\section{FIGURA 4}

PRUEBA DEL RATIO DE VEROSIMILITUD DE QUANDT

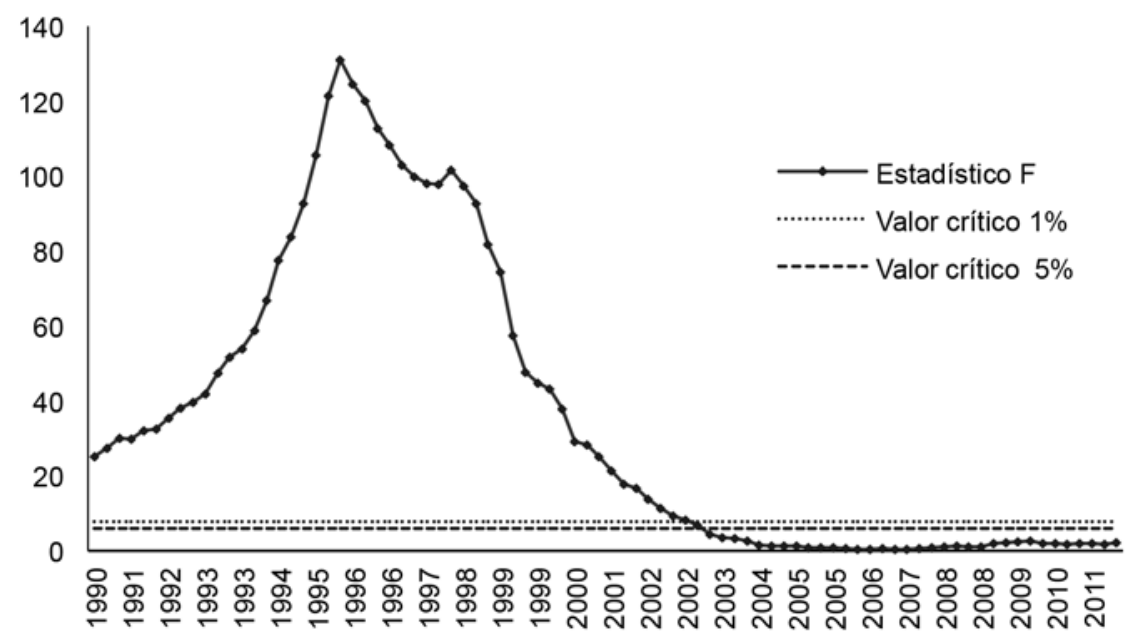

Fuente: Elaboración propia. 
para el modelo con dos restricciones (intercepto y pendiente) al 1 y $5 \%$ de nivel de significancia, alcanzando su valor máximo en el cuarto trimestre de 1995 y se mantiene por encima de los valores críticos hasta el segundo trimestre de 2002. Así, no se puede rechazar la existencia de un posible quiebre estructural.

\subsection{Resultados de la estimación}

Siguiendo la metodología propuesta por Borowczyk-Martins et al. (2013), se estimó el modelo presentado en la especificación (14). Se añadieron adicionalmente tres variables ficticias trimestrales $\left(\tau_{i, t}\right)$ para controlar por efectos estacionales y una variable ficticia $\xi_{t}$ correspondiente al quiebre estructural detectado anteriormente, que toma el valor de 1 para todos aquellos períodos anteriores al segundo trimestre de 2002 y 0 para los períodos posteriores. En consecuencia, el modelo a estimar por MGM es:

$$
f_{t}=\gamma+\sum_{\ell=1}^{p} \rho_{\ell} f_{t-\ell}+\alpha \theta_{t}-\sum_{\ell=1}^{p} \lambda_{\ell} \theta_{t-\ell}+\sum_{i=1}^{3} \beta_{i} \tau_{i, t}+\phi \xi_{t}+\kappa \xi_{t} \theta_{t}+Q(L) \omega_{t}
$$

Aplicando la metodología iterativa expuesta en la sección 3, inicialmente se realizaron estimaciones con el objeto de definir el componente autorregresivo con los valores del grid $p \in(1, \ldots, 6)$. Sin embargo, los estimadores correspondientes a valores de $p$ superiores a 1 resultaron ser estadísticamente no significativos, por lo que se fijó el valor del componente autorregresivo en $p=1$.

A continuación se estimó el modelo con $p$ definido, considerando los valores del grid $q \in(0, \ldots, 6)$. Mediante el análisis del autocorrelograma de residuos de cada estimación se concluyó que el valor más adecuado para el componente de medias móviles es $q=4$. Por tanto, la especificación más adecuada corresponde a un componente $\operatorname{ARMA}(1,4)$. Como se aprecia a la Figura 5, los residuos bajo este modelo muestran un comportamiento puramente aleatorio.

De esta forma, al haber definido la estructura del componente inobservado de la eficiencia del emparejamiento $\epsilon_{t}$ como un proceso $A R M A(1,4)$, la estimación estructural por MGM en este caso descansa sobre los instrumentos con un rezago mayor al proceso $q$, como $\theta_{t-5}, f_{t-5}$ y rezagos superiores; mientras que el intercepto y las variables ficticias trimestrales, al ser exógenas, sirven como instrumentos para sí mismas.

En la Tabla 5 se presentan los resultados de estimación de diferentes especificaciones de la función de emparejamiento. En la columna 1 se incluyen los resultados encontrados por Belani et al. (2002), simplemente para fines comparativos. En dicha investigación por medio de un modelo Vectorial de Corrección de Errores (VCE) se determina que la elasticidad de la rigidez del mercado laboral $\alpha$ es del $16 \%$, es decir, que al incrementarse $\Theta_{t}$ en $1 \%$, la probabilidad de encontrar trabajo se incrementa en $16 \%$. 


\section{FIGURA 5}

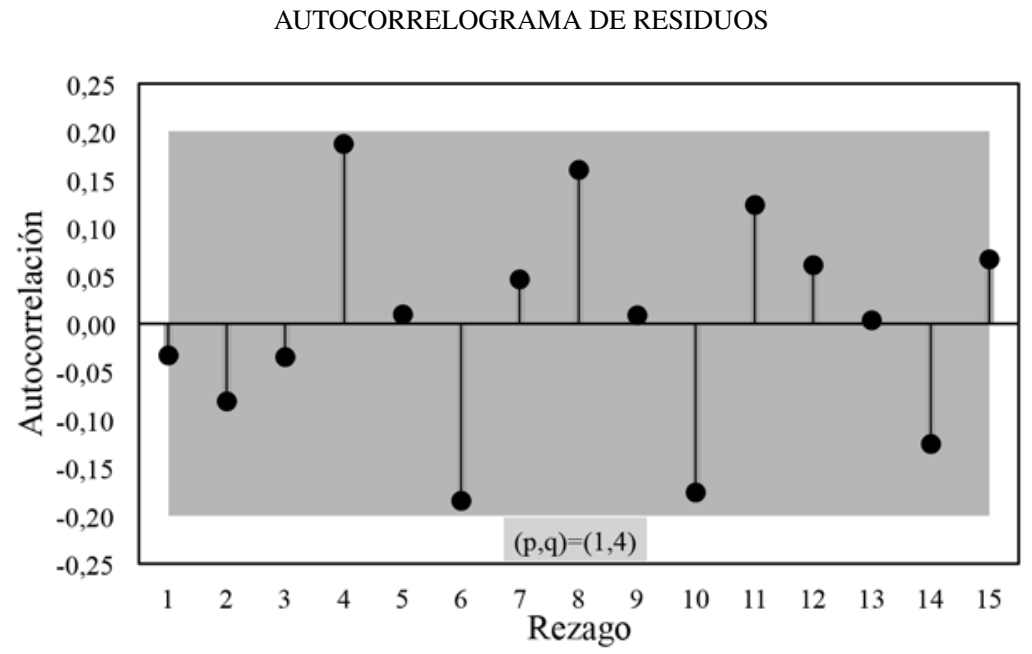

Fuente: Elaboración propia.

Nota: Areas sombreadas corresponden a intervalos de confianza al $90 \%$.

En la columna 2 de la Tabla 5 se observan los resultados de la estimación por Mínimos Cuadrados Ordinarios (MCO), la que como se mencionó anteriormente sufre de sesgos de endogeneidad y problemas de especificación. De nuevo, su inclusión se debió a fines comparativos y evidencia que la elasticidad en este caso es claramente inferior y alcanza $5,49 \%$.

La columna 3 corresponde a la estimación final del modelo propuesto con una identificación exacta, es decir, con un conjunto de instrumentos equivalente al número de variables del modelo. En este caso se registra una elasticidad de la rigidez del mercado laboral de 10,63\% antes del quiebre (segundo trimestre de 2002) y de 11,94\% después del mismo. De acuerdo con el modelo estimado, existe una mayor eficiencia en el emparejamiento en el tercer trimestre de cada año.

Finalmente, la columna 4 muestra los resultados del modelo final sobreidentificado, incluyendo un instrumento adicional al modelo anterior. De acuerdo con esta especificación, la elasticidad de la rigidez del mercado laboral es de $11,68 \%$ en trimestres anteriores al quiebre y de $12,53 \%$ para períodos posteriores. El resto de resultados guardan concordancia con lo obtenido en el modelo anterior. Aunque las estimaciones para ambos modelos demuestran ser robustas, consistentes, así como estadística y económicamente significativas, es evidente que la última especificación gana cierta precisión.

Con el objeto de definir cuál de los modelos propuestos es el más apropiado se considera a continuación los resultados de una prueba de exogeneidad y el diagnóstico 
TABLA 5

RESULTADOS DE ESTIMACION

\begin{tabular}{|c|c|c|c|c|}
\hline & $\begin{array}{c}1 \\
\text { VCE } \\
\text { Belani (2002) }\end{array}$ & $\begin{array}{c}2 \\
\mathrm{MCO}\end{array}$ & $\begin{array}{c}3 \\
\text { MGM } \\
\text { ARMA }(1,4)\end{array}$ & $\begin{array}{c}4 \\
\text { MGM } \\
\text { ARMA }(1,4)\end{array}$ \\
\hline $\begin{array}{c}\gamma \\
\alpha \\
\rho_{1} \\
\beta_{1} \\
\beta_{2} \\
\beta_{3} \\
\phi\end{array}$ & $\begin{array}{c}- \\
- \\
0,1600^{* *} \\
- \\
- \\
- \\
- \\
- \\
- \\
- \\
- \\
- \\
- \\
- \\
- \\
-\end{array}$ & $\begin{array}{c}-0,1514 \\
(0,3309) \\
0,0549 \\
(0,0379) \\
- \\
- \\
-0,1515^{* * *} \\
(0,0379) \\
-0,1659^{* * *} \\
(0,0356) \\
0,0077 \\
(0,0359) \\
1,0368^{*} \\
(0,5389) \\
0,0857 \\
(0,0622)\end{array}$ & $\begin{array}{c}0,0893 \\
(0,1311) \\
0,1194 * * * \\
(0,0335) \\
1,0180 * * * \\
(0,0139) \\
-0,1967 * * * \\
(0,0238) \\
-0,0001 \\
(0,0296) \\
0,1127 * * * \\
(0,0253) \\
-0,1278 \\
(0,1640) \\
-0,0131 \\
(0,0187)\end{array}$ & $\begin{array}{c}0,0623 \\
(0,1311) \\
0,1253^{* * *} \\
(0,0343) \\
1,0390^{* * *} \\
(0,0130) \\
-0,1876^{* * *} \\
(0,0256) \\
0,0031 \\
(0,0269) \\
0,1225^{* * *} \\
(0,0275) \\
-0,0609^{*} \\
(0,0348) \\
-0,0085^{*} \\
(0.0048)\end{array}$ \\
\hline \multicolumn{2}{|c|}{$\begin{array}{l}\text { MGM-Schwarz } \\
\text { MGM-Hannan Quinn } \\
\text { MGM-MSC Relevante }\end{array}$} & $\begin{array}{l}- \\
- \\
-\end{array}$ & $\begin{array}{l}-65.0092 \\
-39.2464 \\
-36,0963\end{array}$ & $\begin{array}{l}-65,5619 \\
-39,7991 \\
-45.8797\end{array}$ \\
\hline \multicolumn{2}{|c|}{$\begin{array}{l}\text { Estadístico } J \text { de Hansen } \\
\text { Valor Probabilidad (Estadístico } J \text { ) }\end{array}$} & - & - & $\begin{array}{l}0,2567 \\
0,6123\end{array}$ \\
\hline
\end{tabular}

Nota: Los instrumentos incluidos en la columna 3 son los rezagos $\theta_{t-\ell}$ para $\ell=q+1=5$ hasta $\ell=q+p+1=6$. Los instrumentos de la columna 4 son iguales a los de la columna 3 añadiéndose $\theta_{t-7}$.

Errores estándar en paréntesis.

* Significancia al $10 \%$.

** Significancia al $5 \%$.

*** Significancia al $1 \%$.

de instrumentos débiles a partir de criterios de selección para modelos estimados por MGM. En el primer caso se considera la prueba de sobreidentificación $J$ de Hansen (1982) (en el caso en que se disponen de más instrumentos que variables explicativas endógenas), que evalúa que los instrumentos cumplan con las condiciones de ortogonalidad necesarias para su empleo. En efecto, la prueba propone la hipótesis nula de que los instrumentos no están correlacionados con el término de error y por esta razón su exogeneidad versus la alternativa de que al menos uno de los instrumentos no es exógeno. 
De acuerdo con lo observado en la Tabla 5, el estadístico $J$ de Hansen para el modelo propuesto en la columna 4 , junto con su valor de probabilidad asociado, indica que no se rechaza la hipótesis nula de que los instrumentos utilizados no están correlacionados con el término de error. Es importante mencionar que también se estimaron modelos alternativos, no reportados en el presente documento, que incluían instrumentos adicionales de los rezagos $\theta_{t}$ y $f_{t}$. Sin embargo, dichas estimaciones resultaron ser muy similares a las expuestas en la Tabla 5 (columnas 3 y 4), con estadísticos $J$ significativamente inferiores a aquellos obtenidos en dicha tabla y en la mayoría de los casos se redujo la significancia estadística de los estimadores. Al respecto, Borowczyk-Martins et al. (2013) indican que debido a la persistencia de $\theta_{t}$ y la correlación entre $f_{t}$ y $\theta_{t}$, instrumentos adicionales no resultan muy informativos y la inclusión de demasiados contribuye a sesgar los resultados.

Por su parte, es importante evaluar la fortaleza de los instrumentos utilizados en un contexto de estimación por variables instrumentales y su generalización por MGM, debido a que instrumentos débiles o marginalmente válidos pueden derivar en una pobre identificación de los parámetros, distribuciones no normales aun en muestras largas, de forma que la inferencia convencional por MGM no es adecuada.

En este sentido, se realiza el diagnóstico de instrumentos débiles a partir de los criterios de selección conocidos como Moment Selection Criteria (MSC), utilizados en las estimaciones por MGM. Se consideran los criterios de Schwarz y HannanQuinn para MGM introducidos por Andrews (1999), análogos a los típicos criterios de selección homónimos. También se considera un tercer criterio conocido como MSC Relevante, introducido por Hall, Inoue, Jana and Shin (2007) de características similares a los anteriores. Al respecto, en la parte inferior de la Tabla 5 se observan los valores de los criterios de selección para los modelos estimados por MGM, donde se aprecia que aquellos correspondientes al modelo de la columna 4 son mucho menores respecto del modelo alternativo, lo que es un indicativo de que el modelo sobreidentificado cuenta con instrumentos más fuertes y por tanto puede brindar una mejor identificación e inferencia. En este sentido el modelo de la columna 4 será considerado como la especificación preferida.

A efectos de interpretación de los resultados, recuérdese que $\alpha$ no solo corresponde a la elasticidad de la rigidez del mercado laboral, sino también a la elasticidad de las vacantes en el flujo de emparejamientos, la que a su vez es entendida como la externalidad positiva de vacantes en desempleados o thick market effect, por lo que los resultados nos indican que, al relajarse la rigidez del mercado laboral en $1 \%$, o equivalentemente, al incrementarse el stock de vacantes en $1 \%$, la probabilidad de encontrar trabajo para un desocupado así como el flujo de emparejamientos se incrementaba en $11,68 \%$ antes del quiebre y en $12,53 \%$ después del mismo.

En este sentido, la reacción de la externalidad positiva de vacantes en desempleados a cambios en el número de vacantes parece haberse incrementado desde inicios del nuevo milenio. Lo mismo se observa en el caso de la eficiencia del emparejamiento. Intuitivamente, se puede deducir que con el tiempo y con el surgimiento y mejora de 
nuevas tecnologías, tanto la externalidad positiva de vacantes en desempleados y la eficiencia del proceso de emparejamiento podrían verse mejoradas, aspecto que se evidencia en los resultados encontrados.

La significancia estadística de casi todos los estimadores para el modelo final es bastante buena, siendo que la elasticidad $\alpha$, los estimadores de los parámetros de los rezagos $\left(f_{t-1}\right.$ y $\left.\theta_{t-1}\right)$, las variables ficticias del primer y tercer trimestre son significativas al $1 \%$. A su vez, los estimadores de los parámetros correspondientes a la variable ficticia de quiebre y su interacción son significativos al $10 \%$.

Como se mencionó anteriormente, los problemas de regresión espuria por temas de no estacionariedad no afectan a la estimación estructural por el Método Generalizado de Momentos. De todas formas, y como prueba de que la relación encontrada no es espuria, se realizó el test de cointegración aumentado de Engle-Granger en los residuos de las distintas especificaciones estimadas. Como se observa en la Tabla 6, para la estimación por Mínimos Cuadrados Ordinarios, aunque el orden de integración de las variables era el mismo, no se puede rechazar la hipótesis de que los residuos sean no estacionarios, incluso considerando el valor crítico al $10 \%$, por lo que dicho modelo no solo sufre del sesgo de endogeneidad, sino que también se trata de una relación espuria y no de cointegración. Sin embargo, en el caso de las dos especificaciones estimadas por MGM, el test muestra que los residuos en ambos modelos son estacionarios.

TABLA 6

TEST ENGLE-GRANGER AUMENTADO

\begin{tabular}{|c|c|c|c|}
\hline Modelo & Estadístico & Valor crítico 5\% & Valor crítico 10\% \\
\hline (2) MCO & $-1,79$ & $-4,70$ & $-4,42$ \\
(3) MGM ARMA $(1,4)$ & $-12,19$ & $-4,70$ & $-4,42$ \\
(4) MGM ARMA $(1,4)$ & $-10,93$ & $-4,70$ & $-4,42$ \\
\hline
\end{tabular}

Fuente: Elaboración propia.

Nota: Valores críticos correspondientes a Mac Kinnon (1994).

\section{CONCLUSIONES}

Debido a la necesidad de contar con una estimación actualizada de la función de emparejamiento o matching function para el mercado laboral chileno y considerando los más recientes avances dentro de la literatura empírica relacionada con dicha estimación, el presente trabajo se constituye en un avance importante al respecto. Aunque en principio la estimación se ve impedida debido a la ausencia de información que sí está disponible en otras economías, como ser el flujo de emparejamientos, las transformaciones llevadas a cabo y los procedimientos utilizados para calcular las 
variables alternativas, como la rigidez del mercado laboral (job market tightness) y la probabilidad de encontrar trabajo (job finding probability), siguiendo a Shimer (2012), permitieron llevar a cabo la estimación de la función de emparejamiento.

Asimismo, los lineamientos tomados en cuenta para realizar la estimación, basados principalmente en el trabajo de Borowczyk-Martins et al. (2013), no solo son importantes debido a la solución del sesgo de endogeneidad presente en este tipo de estimaciones y a la estimación estructural de las elasticidades de interés por medio del Método Generalizado de Momentos, sino también por la consideración de la eficiencia del emparejamiento como un componente variante en el tiempo y sujeto a shocks, algo que resulta intuitivo, pero que se encuentra ausente en la mayoría de las estimaciones de la función de emparejamiento.

Siguiendo al mencionado trabajo, pero considerando también las características propias de los datos utilizados, los mismos que se reflejan en la presencia de un quiebre estructural en el modelo alrededor del 2002, se obtuvo una estimación para la elasticidad de la rigidez del mercado laboral sobre la probabilidad de encontrar trabajo, que a su vez corresponde con la elasticidad del stock de vacantes en el flujo de emparejamientos. Se encuentra que dicha elasticidad alcanza un valor de $11,68 \%$ antes del quiebre y de $12,53 \%$ después del mismo.

Los resultados demuestran ser consistentes, robustos, así como estadística y económicamente significativos y pueden evidenciar que la externalidad positiva de vacantes en desempleados y la eficiencia del proceso de emparejamiento se vieron mejoradas con el tiempo, probablemente debido al surgimiento y mejora de nuevas tecnologías.

\section{BIBLIOGRAFIA}

ALVAREZ P.; F. NUÑEZ y C. USABIAGA (2008). "La Función de Emparejamiento en el Mercado de Trabajo Español”, Revista de Economía Aplicada, Número 48 (Vol. XVI), pp. 5-35.

ANDERSON, P. y S. BURGESS (1995). "Empirical Matching Functions, Estimation and Interpretation using disaggregate data", NBER Working Papers \#5001.

ANDREWS, D. (1999), "Consistent Moment Selection Procedures for Generalized Method of Moments Estimation”, Econometrica, Vol. 67, pp. 543-563.

BARNICHON, R. y A. FIGURA (2015). "Labor Market Heterogeneity and the Aggregate Matching Function", American Economic Journal: Macroeconomics 7 (4), pp. 222-249.

BELANI D.; P. GARCIA y E. PASTEN (2002). "Curva de Beveridge, Vacantes y Desempleo: Chile 19862002.II”, Revista Economía Chilena, Vol. 5 (3), pp. 105-119.

BLANCHARD, O. y P. DIAMOND (1989). “The Aggregate Matching Function”, NBER Working Paper\#3175.

BOROWCZYK-MARTINS D.; G. JOLIVET, y F. POSTEL-VINAY (2013). "Accounting for endogeneity in matching function estimation", Review of Economic Dynamics 16, pp. 440-451.

COLES, M. y B. PETRONGOLO (2008). "A Test Between Stock-Flow Matching and The Random Matching Function Approach", International Economic Review, Vol. 49 No 4, pp. 1113-1141.

COLES, M. y E. SMITH (1998). "Marketplaces and Matching”, International Economic Review, Vol. 39, $\mathrm{N}^{\mathrm{o}} 1$, pp. $239-254$.

FRANSES, P. y B. HOBIJN (1997). "Critical values for unit root tests in seasonal time series", Journal of Applied Statistics, 24 (1), pp. 25-48. 
GALUSCAK, K. y D. MÜNICH (2007). "Structural and Cyclical Unemployment: What Can Be Derived from the Matching Function?", Czech Journal of Economics and Finance 57, pp. 102-125.

HALL A.; A. INOUE, K. JANA y C. SHIN (2007). "Information in generalized method of moments estimation and entropy-based moment selection", Journal of Econometrics, Vol. 138 (2), pp. 488-512.

HANSEN, L. (1982). "Large sample properties of generalized method of moments estimators", Econometrica 50 (3), pp. 1029-1054.

HSIAO, C. (1997). "Statistical Properties of the Two-Stage Least Squares Estimator Under Cointegration", Review of Economic Studies 64, pp. 385-398.

HYLLEBERG S.; R. ENGLE, C. GRANGER y B. YOO (1990). "Seasonal integration and cointegration", Journal of Econometrics, 44 (1), pp. 215-238.

KANO, S. y M. OHTA (2005). "Estimating a matching function and regional matching efficiencies: Japanese panel data for 1973-1999", Japan and the World Economy 17, pp. 25-41.

MACKINNON, J. (1994). "Approximate asymptotic distribution functions for unit-root and cointegration tests", Journal of Business and Economic Statistics, 12, pp. 167-176.

MACKINNON, J. (1996). "Numerical distribution functions for unit root and cointegration tests", Journal of Applied Econometrics, 11, pp. 601-618.

MUJICA, R. (2007). "Descripción crítica de los fundamentos de la Curva de Beveridge y de su uso como herramienta de análisis mercado laboral", Tesis de Magíster, Instituto de Economía, Pontificia Universidad Católica de Chile, <http://economia.uc.cl/docs/tesis_rmujica.pdf>.

PETRONGOLO, B. y C. PISSARIDES (2001). "Looking into the Black Box: A Survey of the Matching Function", Journal of Economic Literature Vol. XXXIX, pp. 390-431.

PISSARIDES, C.A. (1986). "Unemployment and Vacancies in Britain", Economic Policy, Vol. 1, № 3, pp. 499-559.

PISSARIDES, C.A. (2000). "Equilibrium Unemployment Theory”, Segunda Edición. The MIT Press, Cambridge, MA.

SASAKI, M. (2011). "Measuring Matching Efficiency using the public employment service in Japan", Japan Labor Review, Vol. 8, № 3, pp. 78-94.

SHIMER, R. (2003). "The Cyclical Behavior of Equilibrium Unemployment and Vacancies: Evidence and Theory", NBER Working Papers \#9536.

SHIMER, R. (2012). "Reassessing the ins and outs of unemployment", Review of Economic Dynamics 15 , pp. $127-148$.

YASHIV, E. (2000). "The Determinants of Equilibrium Unemployment", American Economic Review December 2000, pp. 1297-1322. 